\title{
Exploring the Choice for Default Systems
}

Frank G. Goethals, IESEG School of Management (LEM-CNRS), Lille, France

\begin{abstract}
This paper investigates the role the default effect plays in choosing IT systems. This paper presents two studies concerning the acceptance of Gmail (vs. a self-hosted mailing system) and of legal (vs. illegal) movie downloading. Classic factors such as the perceived usefulness and ease of use of a system hardly explain the intent to use default systems. Including the perception of an alternative system in the model significantly increases the $\mathrm{R}^{2}$ of the intent to use the default system. Social influence plays a much bigger role in explaining the intent to use default systems than in the intent to use alternative systems.
\end{abstract}

\section{KEYWORDS}

Alternative System, Default Effect, Social Influence, Survey, TAM, Technology Acceptance, Technology Choice, UTAUT

\section{INTRODUCTION}

Few technology acceptance studies have been undertaken where respondents have had to evaluate two substitutive systems. The original Technology Acceptance Model (TAM) paper (Davis, 1989; see literature review) compared acceptance of the Chart-Master system and the Pendraw system. Other seminal papers in this domain assess the acceptance of a single system (Venkatesh et al., 2003; Venkatesh et al., 2012). A wider journal literature review shows researchers systematically focus on acceptance of a single system in isolation. With the exception of one paper (Strader et al., 2007), all TAM papers published in the European Journal of Information Systems since 2007 only studied the acceptance of one system (Van Slyke et al., 2007; Klein, 2007; Neufeld et al., 2007; Bhattacherjee \& Hikmet, 2007; Po-An Hsieh \& Wang, 2007; Khalifa \& Liu, 2007; Dickinger et al., 2008; Sipior et al., 2011; Deng et al., 2010; Stafford \& Hamit Turan, 2011; Yang et al., 2011; Schwarz et al., 2012). This study's primary goal is to assess how acceptance of two substitutive systems are interrelated.

Technology acceptance model studies typically report a relatively high coefficient of determination $\left(\mathrm{R}^{2}\right)$. Technology acceptance papers published since 2006 in Information \& Management - which is the journal that publishes most technology acceptance papers (Bradley, 2012) - give $R^{2} s$ of 0.75 (Hasan, 2006), 0.46 (Cyr et al., 2006), 0.74 (Castaneda et al., 2007), 0.78 (Hsu et al., 2008), 0.60 (Chi et al., 2008), 0.58 (Jin, 2013) and 0.78 (Ho et al., 2015). The lowest $\mathrm{R}^{2}$ reported since 2006 is thus 0.46 (Cyr et al., 2006). The $\mathrm{R}^{2} \mathrm{~s}$ mentioned above for Information \& Management are completely in line with $\mathrm{R}^{2} \mathrm{~s}$ found in other journals. This suggests independent variables in technology acceptance literature explain a large part of the variance in the intention to use every information technology. However, it seems reasonable to believe that little reason exists for researchers who find a low $\mathrm{R}^{2}$ to try to publish papers with little explanatory power, and journals are unlikely to accept papers with a low $\mathrm{R}^{2}$ if no theory is present to explain the low $\mathrm{R}^{2}$. Hence, readers may simply not get the opportunity to see reports of studies with a low $\mathrm{R}^{2}$. 
This article argues a classic application of technology acceptance theory is unlikely to explain much of the variance related to intent to use default systems. The researchers anticipate TAM to provide low $\mathrm{R}^{2}$ in such cases. This study assesses the applicability of the large body of existing technology acceptance theory to explain the use of default systems and alternative systems.

Technology acceptance literature has not yet investigated defaults' role in consumers' IT choices. Partly this is because researchers have wanted to extend TAM and UTAUT (Unified Theory of Acceptance and Use of Technology, Venkatesh et al., 2003) models with context-specific constructs that provide insight into the acceptance of a single specific system. Researchers did not aim to define boundaries round the area where TAM and UTAUT models are highly explanatory. Researchers have tended to add constructs in order to improve those models' validity in specific circumstances (Murphy et al., 2011).

This paper investigates the acceptance of technologies in a voluntary setting. This research follows organisational level theories that suggest similar phenomena. Swanson and Ramiller (2004) have theorised about mindless innovations using IT. A mindless firm doesn't base IT innovations on the organisations' situation. Mindless firms rely on common knowledge in the wider community. Implied endorsement is one of the causes of a default effect (see below). One of the reasons behind mindlessness is the fact that pinpointing the best IT system for a specific company is highly demanding. It then seems acceptable to adopt a default. Organisational members need to consider many concerns (March \& Simon, 1993). They have limited capacity to do so. Hence they may pay attention to more urgent matters, particularly their day-to-day operational responsibilities.

This paper focusses on the consumer's acceptance of technologies. Consumers also tend to have limited time to gather and process information about different systems. In the context of bounded rationality, there is a need to frame classic model applicability such as TAM and UTAUT that require cognitive processing to arrive at a considered solution (van Offenbeek et al., 2013). This paper's second goal is to draw a boundary around the domain in which classic technology acceptance models have high explanatory power on the basis of the default effect theory.

System acceptance in an employee context is outside of the scope of this paper. In contrast to what is happening in an employee context, consumers may want to use several systems with similar functionality (e.g., for e-mail or movie downloading) in parallel. In an employee context, new IT implementations are not always appreciated, and employees often prefer the former system. This suggests that factors leading to the acceptance of the old system lead to a lower acceptance of the new system. This may be the case especially in an employee context, where the company typically supports only system A or system B, but it could also be relevant in a consumer context. Technology acceptance literature in the past started from perceptions concerning system A to explain the acceptance of system A, but the question arises as to whether or not factors driving the acceptance of system B, which could be a substitute for system A, have a negative impact on the acceptance of system A. The researchers' literature review identifies only one paper that has investigated this. Strader et al. (2007, p54) investigate students' intentions to use e-mail and instant messaging and see these as 'two communication technologies that are potentially substitutable'. They hypothesise the perceived usefulness (PU) of system A (B) reduces intent to use system B (A), but their data do not support these hypotheses. That is, their data do not show that the PU of one system is negatively related to the intent to use another system. Strader et al. (2007) note the systems may not be substitutes anyway, for instance because instant messaging is essentially a real-time communication technology, while e-mail is not. As Strader et al. (2007, p55) report that 'previous studies have examined technologies one-at-a-time and thus have not captured potential interdependencies'. This paper's third goal is to assess Strader et al.'s (2007) hypotheses again. This research considers two systems that are more likely to be substitutes. In the first study, the research compares intent to use two different e-mail systems: Gmail vs. a self-hosted mailing system. In the second study, the authors compare intent to use legal vs. illegal channels to download movies. 
16 more pages are available in the full version of this document, which may be purchased using the "Add to Cart" button on the product's webpage:

www.igi-global.com/article/exploring-the-choice-for-defaultsystems $/ 169154$ ?camid $=4 \mathrm{v} 1$

This title is available in InfoSci-Journals, InfoSci-Journal Disciplines Communications and Social Science, InfoSciTechnology Adoption, Ethics, and Human Computer Interaction eJournal Collection, InfoSci-Journal Disciplines Computer Science, Security, and Information Technology. Recommend this product to your librarian: www.igi-global.com/e-resources/libraryrecommendation/?id=2

Related Content

Towards a Theoretical Framework for Creative Participation: How Personal Characteristics Influence Employees' Willingness to Contribute Ideas Natalya Sergeeva and Milan Radosavljevic (2011). Technology for Creativity and Innovation: Tools, Techniques and Applications (pp. 84-103).

www.igi-global.com/chapter/towards-theoretical-framework-creativeparticipation/51985?camid=4v1a

Usability and Player Experience of Input Device for Mobile Gaming Chu Kimberly, Tek Yong Lim, Chee Weng Khong and Chui Yin Wong (2016). International Journal of Mobile Human Computer Interaction (pp. 52-69). www.igi-global.com/article/usability-and-player-experience-of-input-devicefor-mobile-gaming/154076?camid=4v1a

Issues for Australian Indigenous Culture Online Cat Kutay (2011). Handbook of Research on Culturally-Aware Information Technology: Perspectives and Models (pp. 337-361). www.igi-global.com/chapter/issues-australian-indigenous-cultureonline/45049?camid $=4 \mathrm{v} 1 \mathrm{a}$ 
Information Management in Higher Education Administration: A Slow Drive on the Information Superhighway

Gunapala Edirisooriya (2006). Cases on the Human Side of Information Technology (pp. 86-98).

www.igi-global.com/chapter/information-management-higher-educationadministration/6479?camid=4v1a 